\title{
EXTENSIONS AND LOW DIMENSIONAL COHOMOLOGY THEORY OF LOCALLY COMPACT GROUPS. I( $\left.{ }^{1}\right)$ \\ BY \\ CALVIN C. MOORE
}

\section{INTRODUCTION}

Let $A$ be an abelian locally compact( $\left.{ }^{2}\right)$ group and $G$ an arbitrary: locally compact group. We shall say that $A$ is a $G$-module (or a $G$-kernel) if $G$ operates continuously as a group of automorphisms of $A$. By an extension of $G$ by the $G$-module $A$ we shall mean a locally compact group $E$ with a topological isomorphism $i$ of $A$ onto a closed normal subgroup of $E$ and a homomorphismi $j$ of $E$ onto $G$ which induces a topological isomorphism of $E / i(A)$ with $G$ so that the natural operation of $G \simeq E / i(A)$ by conjugation on $i(A) \simeq A$ is the given operation of $G$ on $A$. The notion of equivalence for two such extensions of $G$ by a $G$-kernel $A$ is clear; it is the analogue with the requisite topological restrictions of the notion for finite groups $A$ and $G$.

The familiar notion of the Baer product of two (equivalence classes of) extensions of $G$ by the same $G$-kernel $A$ may be introduced in this context. Hochschild [10] has discussed this operation in the context of Lie groups and it is clear that the discussion applies without change for locally compact groups. With the Baer product the set of equivalence classes of extensions of $G$ by $A$ becomes an abelian group $\operatorname{Ext}(G, A)$. It is the general purpose of this work to try to gain some insight into the global structure of this group of extensions and some of its properties. Many of the theorems are in a sense "finiteness" theorems for these groups. Considered also is the question of endowing the group of extensions with a topology so that it becomes a topological group. It is certainly reasonable to ask if there is any natural way to tell when two extensions of $G$ by $A$ are "close" to one another. Mackey [17] has posed a related question which concerns giving the group of extensions the structure of a Borel group. When the $G$-kernel $A$ is the circle group $T$ with trivial operation by $G$, the group of extensions of $G$ by $T$ is intimately connected with the theory of projective representations of $G$ [19]. The global

Received by the editors June 13, 1961 and, in revised form, May 3, 1963.

(1) This work constitutes in essence part of the author's doctoral thesis at Harvard University, May 1960. Parts were completed under the support of NSF grant G-9504.

(2) We shall use locally compact as an abbreviation for locally compact and separable in the sense of the second axiom of countability. 
structure of the group of extensions of $G$ by $T$ enters into the problem of classifying irreducible representations of locally compact groups.

We shall approach these problems by defining for a locally compact group $G$ and a $G$-kernel $A$, a series $H^{r}(G, A), r \geqq 0$ of cohomology groups of $G$ with coefficients in $A$. These groups are generalizations of the Eilenberg-MacLane groups [5] defined for finite groups $G$. The two dimensional group $H^{2}(G, A)$ is isomorphic to the group of extensions of $G$ by $A$ discussed above. The functoral properties of this cohomology theory and the applicability of many of the ideas of homological algebra will be of the upmost use in our general program. We are, however, not able to obtain very much information about the structure of the higher dimensional cohomology groups.

In the first chapter we define the cohomology groups and note some elementary properties. The latter part of the chapter is devoted to a discussion of an analogue of the spectral sequence of a group extension [12]. We obtain results only in low dimensions but they are adequate for our purposes. In Chapter II, we discuss the low dimensional cohomology groups when $G$ is compact. Theorems $2.1,2.2$ and 2.3 contain the results. In a second paper we shall discuss the situation for noncompact groups and the problem of topologizing the set of group extensions. We also consider there the applications to projective group representations.

Many authors have considered these problems; of particular relevance are papers of Hochschild [10; 11], L. Calabi [3], Shapiro [24], Nagao [22], Bargmann [2], Mostow [27] and others. More important, the results of Mackey [17;18; 19] on group extensions are fundamental to this discussion and have clearly inspired this work. Furthermore it is a pleasure for the author to express his gratitude to Professor Mackey, who was the director for this thesis, for advice and encouragement during its preparation.

We shall fix certain conventions to be used throughout. For a locally compact group $G, \hat{G}$ denotes the set of continuous homomorphisms to the circle group $T$. $T^{n}, R^{n}, Z^{n}$ will as usual denote $n$-fold copies of the circle, line and integers respectively. For an abelian group $A, t(A)$ will denote the torsion subgroup of $A$. We shall adopt the terminology of $[17 ; 18]$ concerning Borel structures. The symbols $\lim d \cdot{ }_{\alpha}$ and $\lim p \cdot{ }_{\alpha}$ will be used to denote the direct and projective (inverse) limits respectively of a system of groups $G_{\alpha}$. The numbers in brackets refer to the bibliography at the end.

\section{CHAPTER I}

1. In this chapter we shall define and prove some general properties of the cohomology groups which we propose to study. It is desirable to enlarge the scope of definition of these groups in order to place them in a more natural context.

Definition 1.1. A Borel group $G$ (cf. [9, p. 257; 18]) is an abstract group $G$ with a $\sigma$-field of subsets $\Sigma$ of $G$ so that $\{p\} \in \Sigma$ for all $p \in G$, and so that the map $(x, y) \rightarrow x y^{-1}$ of $G \times G$ to $G$ is a Borel function. 
If $A$ is an abelian Borel group and $G$ any Borel group, then $A$ is a $G$-module when $A$ is defined as a $G$-module purely algebraically by a map $(s, a) \rightarrow s \cdot a$ of $G \times A$ to $A$, and so that in addition $s \cdot a$ is a Borel function from $G \times A$ to $A$. It is evident that we may speak of the category [7, p. 11] of $G$-modules; homomorphisms from $A$ to $B$ are required to be Borel functions and to commute with the operation of $G$. However, it does not seem to be possible to make this an abelian category and thus to make available an axiomatic definition of cohomology groups. The difficulty can be attributed to the presence of "bad" subgroups $H$ in some Borel groups $G$ for which $G / H$ does not have very much sense (such as a line $R$ embedded densely in a torus). The following definition singles out a useful regularity property.

Definition 1.2. A subgroup $H$ of a Borel group $G$ is closed if there is a subset $K$ of $G$ so that the projection $G \rightarrow G / H$ (right cosets), when restricted to $K$ is a Borel isomorphism of $K$ with $G / H$ (taken with the quotient Borel structure).

It is easy to see that $K$ may be chosen so that $K \cap H$ consists of the identity of $G$. The following facts about closed subgroups are easily verified.

Proposition 1.1. If $H$ is a closed subgroup of $G$, then $H \times K \approx G$ as Borel spaces; the isomorphism is effected by $(h, s) \rightarrow h \cdot s$. If $H$ is a closed normal subgroup, $G / H$ is a Borel group in the quotient Borel structure.

We shall with the aid of this definition introduce into the category of $G$-modules a notion of exact sequence.

Definition 1.3. If $A^{\prime}, A, A^{\prime \prime}$ are $G$-modules, then we say that

$$
0 \rightarrow A^{\prime} \stackrel{i}{\rightarrow} A \stackrel{j}{\rightarrow} A^{\prime \prime} \rightarrow 0
$$

is an exact sequence when:

(1) It is algebraically exact as algebraic $G$-modules.

(2) $i$ and $j$ are Borel $G$-homomorphisms and $i$ is a Borel isomorphism of $A^{\prime}$ onto the subgroup $B$ of $A$ which is its range; $B$ is a closed subgroup $B$ of $A$ which is its range; $B$ is a closed subgroup of $A$ and $j$ induces a Borel isomorphism of $A$ / $B$ with $A^{\prime \prime}$.

With these preliminaries completed, we turn to the definition of the cohomology groups. We shall make appropriate modifications of the Eilenberg-MacLane theory prompted by the considerations above; these ideas are clearly inspired by the work of Mackey [17]. If $A$ is a $G$-module, then the normalized cochain group is defined as $C^{n}(G, A)=\{f: f$ is a Borel function from $G \times \cdots \times G$ ( $n$ factors) to $A$, and $f\left(s_{1}, \cdots, s_{n}\right)=0$ if some $s_{i}=e$ in $\left.G\right\}$ for $n \geqq 0 ; C^{n}(G, A)=0$ for $n<0$. The coboundary operator $\delta_{n}$ is defined as usual for $f \in C^{n}(G, A) ; \delta_{n} f\left(s_{1}, \cdots, s_{n+1}\right)$ $=s_{1} \cdot f\left(s_{2}, \cdots, s_{n+1}\right)+\sum_{i=1}^{n}(-1)^{i} f\left(s_{1}, \cdots, s_{i} s_{i+1}, \cdots, s_{n+1}\right)+(-1)^{n+1} f\left(s_{1}, \cdots, s_{n}\right)$.

Proposition 1.2. (1) $\delta_{n}$ maps $C^{n}(G, A)$ into $C^{n+1}(G, A)$.

(2) $\delta_{n+1} \delta_{n}=0$. 
Proof. These assertations follow almost by analogy with known results. The only point to be noticed is that $\delta_{n} f$ is indeed a Borel function of its $n+1$ variables. To see this, it suffices to check if for each term of the expression for $\delta_{n} f$. The first term is a Borel function since $(s, a) \rightarrow s \cdot a$ is a Borel function; for the remaining terms, we observe that $\left(s_{i}, s_{i+1}\right) \rightarrow s_{i} s_{i+1}$ is a Borel function from $G \times G$ to $G$.

The cohomology groups $H^{n}(G, A)$ of the complex $C^{*}(G, A)$ are formed as usual. The mapping properties of these cohomology groups parallel those of the Eilenberg-MacLane groups (to which these groups clearly reduce when $G$ is finite). If $\alpha$ is a Borel homomorphism of $G^{\prime}$ into $G$ and $j$ a compatible Borel homomorphism of the $G$-module $A$ to the $G^{\prime}$-module $A^{\prime}$ (i.e., $s^{\prime} \cdot j(a)=j\left(\alpha\left(s^{\prime}\right) \cdot a\right)$ for $s^{\prime} \in G^{\prime}$, $a \in A$ ) then we deduce a homomorphism of complexes $C^{*}(G, A) \rightarrow C^{*}\left(G^{\prime}, A^{\prime}\right)$. In dimension $n, f^{\prime}\left(s_{1}^{\prime}, \cdots, s_{n}^{\prime}\right)=j\left(f\left(\alpha\left(s_{1}^{\prime}\right), \cdots, \alpha\left(s_{n}^{\prime}\right)\right)\right) ; f^{\prime}$ is a Borel function since $\alpha, j$ and $f$ are. The corresponding maps on cohomology are analogous to those of the Eilenberg-MacLane theory. We have a restriction map if $G^{\prime}$ is a subgroup of $G$ and an inflation map if $G^{\prime} \approx G / H$ where $H$ is a closed normal subgroup. Also for fixed $G, H^{2}(G, A)$ are covariant functors of $A$. We turn now to the crucial analogue of the exactness property of cohomology groups for finite groups.

Proposition 1.3. If $0 \rightarrow A^{\prime} \stackrel{i}{\rightarrow} A \stackrel{j}{\rightarrow} A^{\prime \prime} \rightarrow 0$ is an exact sequence of $G$-modules (Definition 1.3), then

$$
0 \rightarrow C^{*}\left(G, A^{\prime}\right) \stackrel{\hat{\imath}}{\rightarrow} C^{*}(G, A) \stackrel{\hat{\jmath}}{\rightarrow} C^{*}\left(G, A^{\prime \prime}\right) \rightarrow 0
$$

is an exact sequence of complexes.

Proof. That $\hat{\imath}$ is an injection and that $\hat{j} \circ \hat{\imath}=0$ are trivial. If $f \in C^{*}(G, A)$ belongs to $\operatorname{ker}(\hat{\jmath})$ then $f$ takes values in $i\left(A^{\prime}\right)$ and since $i^{-1}$ is a Borel map of $i\left(A^{\prime}\right)$ to $A^{\prime}, i^{-1} f$ belongs to $C^{n}\left(G, A^{\prime}\right)$ and $\hat{\imath}$ applied to it is $f$. Thus $\operatorname{ker}(\hat{\jmath})=\operatorname{Im}(\hat{\imath})$. Finally, if $f \in C^{n}\left(G, A^{\prime \prime}\right)$, then since $i\left(A^{\prime}\right)$ is a closed subgroup, there is by Definitions 1.2 and 1.3 a Borel isomorphism $\phi$ of $A^{\prime \prime}$ into $A$ so that $j \phi=i d$, and so that $\phi\left(0^{\prime \prime}\right)=0$. The function $g=\phi f$ is then a normalized Borel cochain of $G$ in $A$ of dimension $n$. We have then $\hat{j} g=f$ since $j \phi=i d$. Thus $\hat{\jmath}$ is surjective. Q.E.D.

Consequently from the well-known theorem $[7$, p. 20] we deduce the existence of the usual infinite exact sequence of cohomology

$$
\stackrel{\partial_{n}^{-1}}{\longrightarrow} H^{n}\left(G, A^{\prime}\right) \stackrel{i^{*}}{\longrightarrow} H^{n}(G, A) \stackrel{j^{*}}{\longrightarrow} H^{n}\left(G, A^{\prime \prime}\right) \stackrel{\partial_{n}}{\longrightarrow} H^{n+1}\left(G, A^{\prime}\right) \longrightarrow .
$$

The groups $H^{n}(G, A)$ for $r=0,1,2$ have interpretations analogous to these groups when $G$ is finite. It is clear that $H^{0}(G, A)$ is $A^{G}$ (the elements in $A$ left fixed by the operation of $G) \cdot H^{1}(G, A)$ is similarly isomorphic to the group of all Borel crossed homomorphisms of $G$ into $A$ modulo the principal ones (i.e., of the form $f(s)=s \cdot a-a$ for some $a \in A)$. Finally $H^{2}(G, A)$ is isomorphic to a group of classes of extensions. This cohomology group classifies Borel groups $E$ which contain a Borel isomorphic copy $i(A)$ of $A$ as a closed normal subgroup, 
and which have a projection $j$ of $E$ onto $G$ so that $j$ sets up a Borel group isomorphism of $E / i(A)$ with $G$, and finally subject to the condition that the operation of $G$ on $A$ by inner automorphisms of $E$ is just the given operation of $G$ on the $G$-module $A$. Equivalence of such groups $E$ is defined in the obvious way. That $H^{2}(G, A)$ does classify such extensions can be proved by an analogue of the wellknown argument in the case when $G$ is finite. The fact that $i(A)$ is closed in $E$ and Proposition 1.1 provide the key to showing that these arguments can be carried out so that the resulting functions (factor sets, etc.) are Borel functions. We shall omit the details.

2. The results of the previous section supply only a context for the cohomology theory. Their significance lies in their interpretation when $G$ and $A$ are locally compact. For if $A$ is a locally compact $G$-module ( $G$ locally compact) as described in the introduction, then $A$ and $G$, given the Borel structure generated by the open sets, are Borel groups and $A$ is a Borel $G$-module. Conversely, we have the following:

Proposition 1.4. If $A$ and $G$ are locally compact, and $A$ is a Borel G-module, then it is a topological G-module.

Proof. The action of $G$ on $A$ by $(s, a) \rightarrow s \cdot a$ is assumed to be a Borel function ; we must show that it is continuous. The reader will recognize this argument as a slight variant of an argument given by Banach [1, p. 25].

(a) Fix $s_{0} \in G$; then $a \rightarrow s_{0} \cdot a$ is by restriction a Borel automorphism of $A$. Hence by Banach's theorem $[1$, p. $25 ; 15$, p. 399] it is continuous.

(b) Fix $a_{0} \in A$; then $s \rightarrow s \cdot a_{0}$ is a Borel function from $G$ to $A$. Since $A$ is separable, it is known $[15$, p. 307$]$ that there is a set $P$ of the first category so that this function restricted to $G-P$ is continuous. Let a sequence $s_{n} \rightarrow s_{0}$ be given in $G$. Since $\bigcup_{n=0}^{\infty} P s_{n}^{-1}$ is of the first category in $G$, there is a point $s \in G$ not in this set ( $G$ is a complete metric space and so of the second category). Thus $s s_{n} \notin P$ for $n=0,1, \cdots$, and $s s_{n} \rightarrow s \cdot s_{0}$. We conclude that $s s_{n} \cdot a_{0} \rightarrow s s_{0} \cdot a_{0}$, and since the operation of $s^{-1}$ on $A$ is continuous by part (a), we see finally that $s_{n} a_{0} \rightarrow s_{0} a_{0}$. Then $s \rightarrow s \cdot a_{0}$ is continuous.

(c) Using these two partial results, we may now prove the proposition. Let $Q$ be a set of the first category in $G \times A$ so that $s \cdot a$ is continuous when restricted to $G \times A-Q$. Let $\left(s_{n}, a_{n}\right) \rightarrow\left(s_{0}, a_{0}\right)$; as in part (b) we may find a point $(s, a)$ so that $\left(s s_{n}, a+a_{n}\right) \notin Q$ for $n=0,1, \cdots$. Then as above $s s_{n}\left(a+a_{n}\right) \rightarrow s s_{0}\left(a+a_{0}\right)$. If we now apply the operation of $s^{-}$to this (part (a)), and then subtract the convergence relation $s_{n} a \rightarrow s_{0} a$ (part (b)), we can conclude that $s_{n} a_{n} \rightarrow s_{0} a_{0}$. This completes the proof.

We shall now interpret the notions of closed subgroups and exact sequences of $\S 1$ in the context of locally compact groups. This will serve also to justify the usual terminology of "closed." 
Proposition 1.5. (1) If $G$ is locally compact, a subgroup $H$ is closed (Definition 1.2) iff $H$ is topologically closed in $G$.

(2) If $A^{\prime}, A, A^{\prime \prime}$ in Definition 1.3 are locally compact, the sequence is exact iff it is algebraically exact, and $i$ and $j$ are continuous.

Proof. Part (1) is just Lemma 1.1 [16] and Theorem 7.2 [18]. For the necessity in part (2), we observe that $i, j$ being Borel homomorphisms, are continuous. For the sufficiency, we observe that since $j$ is continuous, $\operatorname{ker}(j)=\operatorname{Im}(i)$ is topologically closed, hence closed in the sense of Definition 1.2. Now $i\left(A^{\prime}\right)$ is locally compact, and by the closed graph theorem [15, p. 399] $i$ is a homeomorphism, hence a Borel isomorphism. Finally $j$ induces a continuous map $j^{\prime}$ of $A / i\left(A^{\prime}\right)$ onto $A^{\prime \prime}$. By [15, p. 397], $j$ is a Borel isomorphism, and this completes the proof.

If $G$ and $A$ are locally compact and $A$ is a $G$-module, the cohomology groups $H^{n}(G, A)$ are those defined in $\S 1$, with $G$ and $A$ viewed as Borel groups. Their properties are direct consequences of $\S 1$ and Proposition 1.5. The group $H^{0}(G, A)$ is of course $A^{G}$ where $A^{G}$ is a closed subgroup; the group $H^{1}(G, A)$ is as before, but we now contend that a Borel crossed homomorphism is necessarily continuous. Another variant of Banach's argument (cf. Proposition 1.4) can be used to prove this fact. Thus $H^{1}(G, A)$ consists of continuous crossed homomorphisms modulo the principal ones. As remarked in the introduction, our approach to the extension problem is to interpret the group of extensions $\operatorname{Ext}(G, A)$ described there as a cohomology group. That indeed the group $H^{2}(G, A)$ defined in this section is isomorphic to the group $\operatorname{Ext}(G, A)$ for a locally compact $G$-module $A$ as described in the introduction has been proved by Mackey [17]. Thus the cohomology groups defined above for locally compact groups generalize many of the properties of the Eilenberg-MacLane groups. The low dimensional groups have in a sense the proper interpretations in the terms of homomorphisms and extensions, and the theory as a whole has many of the mapping or functoral properties which they possess if $G$ is finite. In the next section we will press this further to obtain an analogue of the spectral sequence of a group extension of [12].

Before doing so, we concern ourselves with a useful variant of the cohomology groups just defined when $G$ and $A$ are locally compact. The subgroup $\bar{C}^{n}(G, A)$ of $C^{n}(G, A)$ will consist of all cochains $f$, bounded on bounded sets in $G \times \cdots \times G$. (A set $B$ is bounded if its closure $\bar{B}$ is compact.) A slight extension of Proposition 1.2 shows that $\delta_{n} f$ is bounded on bounded sets if $f$ is. Thus the groups $\bar{C}^{n}(G, A)$ together with the restrictions of the $\delta_{n}$ form a complex; the cohomology groups will be denoted by $\bar{H}^{n}(G, A)$ (bounded cohomology groups of $G$ with coefficients in $A$ ). The properties of the groups $H^{n}(G, A)$ in $\S 1$ extend without difficulty to the bounded groups. The only point of significance is the "lifting" process in Proposition 1.3. In that notation it need not be so that the result of lifting $f$ from $A^{\prime \prime}$ to $A, g=\phi(f)$ is bounded on bounded sets even if $f$ is. If, however, we choose 
the cross section of $A^{\prime \prime}$ in $A$ according to the following proposition, the difficulty is avoided.

Proposition 1.6. If $G$ is locally compact and $H$ a closed subgroup, there is a cross section $K$ of $G / H$ in $G$ having the properties of Definition 1.2 and so that if $C$ is a bounded set in $G / H$, the intersection of $K$ with its counterimage in $G$ is bounded.

Proof. This is proved by Mackey [16, Lemma 1.1].

The inclusion map of $\bar{C}^{n}(G, A)$ into $C^{n}(G, A)$ is a homomorphism of complexes and so induces maps $\alpha_{n}$ from $\bar{H}^{n}(G, A)$ into $H^{n}(G, A)$. A significant relation between the bounded cohomology groups and the ordinary groups is summarized in the following proposition.

Proposition 1.7. The homomorphisms $\alpha_{n}$ are isomorphisms in dimensions $n=0,1,2$.

Proof. The assertion is clear in dimension zero; in dimension one, since we have remarked that a Borel crossed homomorphism is continuous, such a map is clearly bounded on bounded sets. Thus $Z^{1}=Z^{1}$. Moreover $B^{1}=\bar{B}^{1}$ since $C^{0}=\bar{C}^{0}$. Then $\alpha_{1}$ is evidently an isomorphism.

Finally let $\beta \in H^{2}(G, A)$; then $\beta$ defines a (class of) extensions of $G$ by $A$. If $E$ is such an extension, let $K$ be a cross section of $G$ in $A$ satisfying the condition of Proposition 1.6, and let $\psi$ be the corresponding map of $G$ onto $K \subseteq E$. The function

$$
b(s, t)=\psi(s) \psi(t) \psi(s t)^{-1} \in A \subseteq E
$$

is a cocycle representing $\beta$. The function $\psi$ maps bounded sets into bounded sets. If $(s, t)$ range over a bounded set in $G, \psi(s), \psi(t)$ and $\psi(s t)^{-1}$ range over bounded sets in $E$. Thus likewise $b(s, t)$ is bounded on bounded sets. Since $\beta$ now contains an element of $Z^{2}$, it is clear that $\alpha_{2}$ is surjective.

To show that $\alpha_{2}$ is injective, we must show that if $a \in Z^{2}$ and $a=\delta_{1} \gamma$ for some $\gamma \in C^{1}$, then $a=\delta_{1} \gamma^{1}$ for some $\gamma^{1} \in \bar{C}^{1}$. In fact, it is true that $\gamma \in \bar{C}^{1}$ already. For suppose to the contrary; then there is a bounded sequence $s_{n}$ in $G$ so that $\gamma\left(s_{n}\right)$ is unbounded. We may and shall suppose that $s_{n}$ converges to some $s_{0}$. As we have argued before, there is a set $P$ of the first category in $G$ so that the Borel function $\gamma$ is continuous when restricted to $G-P$. Also there is a point $t \in G$ so that $s_{n} t \notin G-P$ for all $n$. Rewriting $a=\delta_{1} \gamma$, we have

$$
\gamma\left(s_{n}\right)=a\left(s_{n}, t\right)-s_{n} \gamma(t)+\gamma\left(s_{n} t\right) .
$$

The first term on the right is bounded by hypothesis; the second and third terms converge to $s_{0} \cdot \gamma(t)$ and $\gamma\left(s_{0} t\right)$ respectively. The right-hand side of the above is then bounded as $n$ tends to infinity, but the left-hand side is not. This contradiction completes the proof. 
With this proposition in mind we shall in some cases use $H^{2}(G, A)$ and $\bar{H}^{2}(G, A)$ interchangeably. Of course, when $A$ is compact as it often will be in applications there is no difference at all in any dimension. The reason for the introduction of the bounded groups $\bar{H}^{n}$ is that in many cases they have useful properties sometimes not shared by the groups $H^{n}$ and conversely.

3. We shall now construct one of our principal tools, the spectral sequence of a group extension. It is constructed in terms of the ordinary theory; for the bounded theory, one would proceed simply by imposing the boundedness conditions at the proper instances. Let then $G$ be locally compact, $H$ a closed normal subgroup of $G$, and $A$ a locally compact $G$-module. In the ensuing discussion, we shall follow exactly the treatment of this subject by Hochschild and Serre ([12, Chapter II], abbreviated as H-S), making the necessary amendment and remarks to show that their arguments can be carried over to our Borel cohomology.

Before proceeding with this we shall try to relate it to some known facts. The spectral sequence will introduce on $H^{2}(G, A)=K_{0}$, a three step filtering, $K_{0} \supset K_{1} \supset K_{2} \supset 0 . K_{1}$ is the kernel of the restriction map from $K_{0}$ to $H^{2}(H, A)^{G / B}$ and $K_{2}$ is the image of the inflation map from $H^{2}\left(G / H, A^{H}\right)$. Moreover there is an injective homomorphism of $K_{1} / K_{2}$ into $H^{1}\left(G / H, H^{1}(H, A)\right)$. In the special case when $A$ is the circle group $T$, with $G$ operating trivially, and when $G$ is a semi-direct extension of $G / H$ by $H$, Mackey $[19$, pp. $303 \mathrm{ff}$.] has proved such a result without the terminology of spectral sequences. In one respect the spectral sequence simply removes these restrictions, and one could make a direct construction of the filtering and homomorphisms along the lines of Mackey's result and thus avoid the complicated construction to follow. This is sufficient for some but not all of our purposes; the finer properties of a spectral sequence are needed such as the higher order derivatives $d_{2}, d_{3}$, the filtering in higher dimensions and the relations between the finite terms $E_{r}$ and the $E_{\infty}$ term of the spectral sequence.

We introduce a filtering $\left\{L_{j}\right\}$ on the complex $C^{*}(G, A)$ as follows:

(1) $L_{j}=\sum_{n=0}^{\infty}\left(L_{j} \cap C^{n}(G, A)\right)$.

(2) $L_{j} \cap C^{n}(G, A)=\left\{f \in C^{n}(G, A)\right.$ : the value of $f\left(s_{1}, \cdots, s_{n}\right)$ depends on the first $n-j$ arguments $s_{1}, \cdots, s_{n-j}$, but only on the $H$-cosets of the final $j$ arguments if $0 \leqq j \leqq n$.

(3) Finally $L_{j} \cap C^{n}(G, A)=0$ for $j>n$ and $L_{j}=C^{*}(G, A)$ for $j<0$.

It is purely formal that this filtering is compatible with differentiation; i.e., $\delta L_{j} \subset L_{j}$. The filtration is positive and regular [7,pp. $70 \mathrm{ff}$.], and it follows that this filtering defines a spectral sequence $E_{r}^{j i}$ which abuts to $H^{*}(G, A)$ appropriately filtered. Also $E_{r}^{j i}=0$ unless $j, i \geqq 0$.

When $G$ is finite, H-S determine the $E_{2}$ term of this spectral sequence as $\mathrm{E}_{2}^{j i} \simeq H^{j}\left(G / H, H^{i}(H, A)\right)$. In the present situation the groups on the right have no meaning since $H^{j}(G / H, B)$ is defined only when $B$ is a Borel group, and 
$H^{i}(H, A)$ has as yet no Borel structure (the cases $i=0,1$ will be fortunate exceptions). The question mentioned earlier of assigning a suitable topology to the group of extensions $H^{2}(H, A)$ is of course crucial to the problem of extending the results that we do obtain (Theorem 1.1). We approach these difficulties by making the following definition.

Definition 1.4. $C^{j}\left(G / H, C^{i}(H, A)\right)=\{f: f$ is a normalized $j$-cochain on $G / H$ with values in $C^{i}(H, A)$ subject to the condition that the function $f$ defined by $f^{f}\left(h_{1}, \cdots, h_{i}, \hat{s}_{1}, \cdots, \hat{s}_{j}\right)=\left[f\left(\hat{s}_{1}, \cdots, \hat{s}_{j}\right)\right]\left(h_{1}, \cdots, h_{i}\right)$ is a Borel function of its $i+j$ variables ( $i$ of them in $H, j$ of them in $G / H$ ) $\}$.

It is clear that $C^{j}\left(G / H, C^{i}(H, A)\right)$ is a group under the natural addition; moreover the differentiations $\delta_{H}^{*}$ of the complex $C^{*}(H, A)$ induce map $\delta_{H}^{j i}$ of $C^{j}\left(G / H, C^{i}(H, A)\right)$ into $C^{j}\left(G / H, C^{i+1}(H, A)\right)$. That $\delta_{H}^{j i} f$ does indeed satisfy the Borel condition of Definition 1.4 if $f$ does is evident. We denote the $i$ th cohomology group of the complex so formed by $H^{i}\left(C^{j}\left(G / H, C^{*}(H, A)\right)\right)$.

Following H-S, we observe that the term $E_{1}^{j i}$ of our spectral sequence is isomorphic to $H^{i+j}\left(L_{j} / L_{j+1}\right)$. If $f \in L_{j} \cap C^{i+j}(G, A)$, define $r_{j}^{\prime}(f)$ to be the element of $C^{j}\left(G / H, C^{i}(H, A)\right.$ given by $\left(r_{j}^{\prime} f\left[\hat{s}_{1}, \cdots, \hat{s}_{j}\right]\right)\left(h_{1}, \cdots, h_{i}\right)=f\left(h_{1}, \cdots, h_{i}, s_{1}, \cdots, s_{j}\right)$. (The symbol $h$ denotes a generic element of $H$ and $\hat{s}$ denotes the image of $s \in G$ in $G / H$.) That the formula has sense and that $r_{j}^{\prime} f$ satisfies the condition of Definition 1.4 is again clear. If $f$ in addition belongs to $L_{j+1}$, then $f\left(h_{1}, \cdots, h_{i}, s_{1}, \cdots, s_{j}\right)=0$ since the value depends only on the coset of $h_{i}=(H e)$. Therefore $r_{j}^{\prime}$ induces a homomorphism $r_{j}$ of $L_{j} \cap C^{i+j}(G, A) / L_{j+1} \cap C^{i+j}(G, A)$ into $C^{j}\left(G / H, C^{i}(H, A)\right)$. If $\delta^{j}$ denotes the differentiation of the complex $L_{j} / L_{j+1}$ (graded by the images of $C^{i+j}(G, A)$ ), it is purely formal to verify that $r_{j}$ is a homomorphism of complexes. The map $r_{j}$ induces a homomorphism $r_{j}^{*}$ on cohomology; our first result, the analogue of Theorem 1, p. 121 of $\mathrm{H}-\mathrm{S}$ is contained in the following:

LEMMA 1.1. The homomorphism $r_{j}^{*}$ is an isomorphism of $H^{i+j}\left(L_{j} / L_{j+1}\right)$ $\simeq E_{1}^{j i}$ with $H^{i}\left(C^{j}\left(G / H, C^{*}(H, A)\right)\right)$ for all $i$.

Proof. The argument given in Theorem 1 of $\mathrm{H}-\mathrm{S}$ applies formally to this situation once we can establish that the constructions made there can be performed in the present context so as to preserve the Borel conditions which we impose on cocycles. We first choose a cross section $N$ of $G / H$ in $G$ subject to the conditions of Proposition 1.5. Let the correspondence $\hat{s} \rightarrow \hat{s}^{*}$ denote the selection of coset representatives defined by $N$. The crux of the argument in $\mathrm{H}-\mathrm{S}$ is the construction from a set of data of a family of functions $g_{k}, k=0, \cdots, i$, where $g_{k}$ is a function of $i+j$ variables, the first $k$ and final $j$ of which range over $G$ and the others from $H$. We contend that with the choice of coset representatives $\hat{s}^{*}$, the functions $g_{k}$ defined by H-S are Borel functions of their $i+j$ variables.

The function $g_{0}$ is given initially as a Borel function; then 


$$
g_{1}\left(\hat{s}^{*} h_{1}, h_{2}, \cdots, h_{i}, s_{1}, \cdots s_{j}\right)=\hat{s}^{*} g_{0}\left(h_{1}, \cdots, h_{i}, s_{1}, \cdots, s_{j}\right)-f\left(\hat{s}^{*}, h_{1}, \cdots, h_{i}, s_{1}, \cdots, s_{j}\right)
$$

where $f$ is some preassigned element of $C^{i+j+1}(G, A)$. Since the 'components' $\hat{s}^{*}$ and $h_{1}$ of $s=\hat{s}^{*} h_{1}$ are Borel functions of $s$, it becomes manifest that $g_{1}$ is a Borel function. Proceeding analogously we can show that all the $g_{k}$ are Borel functions; for $k=i$, we conclude that $g_{i}$ belongs to $C^{i+j}(G, A)$. With this observation we are finished, for the rest of the argument is purely formal, and applies without change.

The cycles $Z^{j i}$ in dimension $i$ of the complex $C^{j}\left(G / H, C^{*}(H, A)\right)$ can be easily identified, for $f$ is such a cycle if it satisfies the Borel condition laid down in Definition 1.4, and if as a function from $(G / H)^{j}$ to $C^{i}(H, A)$, it assumes its values in $Z^{i}(H, A)$. The boundaries $B^{j i}=\operatorname{Im}\left(\delta_{H}^{j, i-1}\right)$ make up a subgroup of $Z^{j i}$ of functons which assume values in $B^{i}(H, A)$; however, there seems to be no reason in general why $B^{j i}$ should consist of all functions in $Z^{j i}$ which take values in $B^{i}(H, A)$. Even if this could be proved so that $H^{j i}$ could plausibly be thought of as $C^{j}\left(G / H, H^{i}(H, A)\right)$, we are faced with a second and further problem of assigning $H^{i}(H, A)$ a Borel structure so that $H^{j i}$ coincides exactly with the class of Borel functions from $(G / H)^{j}$ to $H^{i}(G, A)$.

In several trivial cases these difficulties do not occur; (1) when $i=0$, $B^{0}(H, A)=0$ and it is clear that $E_{1}^{j 0} \simeq C^{j}\left(G / H, A^{H}\right) ;(2)$ when $j=0$, $C^{0}\left(G / H, C^{i}(H, A)\right)$ is just $C^{i}(H, A)$ so that $E_{1}^{0 i} \simeq H^{i}(H, A)$. The following definition is easily seen to be aimed at the general resolution of the first problem mentioned above. Despite its complexity and ad hoc nature, it turns out that it can be readily verified in many interesting cases.

Definition 1.5. The complex $C^{*}(H, A)$ is regular in dimension $i>0$ if there is a map $\phi_{i}$ of $B^{i}(H, A)$ into $C^{i-1}(H, A)$ so that

(1) $\delta_{H}^{i-1} \phi_{i}=i d$.

(2) If $E$ is any Borel space and $f$ is any Borel map of $E \times H^{i}$ into $A$ so that $f(s, \cdot) \in B^{i}(H, A)$ for all $s \in E$, then $\phi_{i}(f(s, \cdot))$ is a Borel function when viewed as map from $E \times H^{i-1}$ into $A$.

If $C^{*}(H, A)$ is regular in dimension $i$, then $r_{j}^{*}$ induces an isomorphism of $E_{1}^{j i}$ with $\widetilde{C}^{j}\left(G / H, H^{i}(H, A)\right)$ where we define this last group to be $Z^{j i}$ (see above) modulo those functions in $Z^{j i}$ which assume values in $B^{i}(H, A)$. When $C^{*}(H, A)$ is regular in dimension $i$, the argument of H-S, p. 123, applies almost directly to determine the $E_{2}$ term of our spectral sequence in complementary degree $i$.

The partial coboundary operators $\delta_{i}$ and $\partial_{j}(\mathrm{H}-\mathrm{S}, \mathrm{p} .123)$ operate on $C^{i+j-1}(G, A)$ for it is evident by familiar arguments that $\delta_{i} f$ and $\partial_{j} f$ are Borel functions of $i+j$ variables if $f \in C^{i+j-1}(G, A)$. The same is evidently true of the shuffling operators $g \rightarrow g_{s}$. Finally Proposition 2, being purely algebraic, remains valid. We must define a differentiation on the family of groups $\mathcal{C}^{j}\left(G / H, H^{i}(H, A)\right)$. If an element $F$ of the latter group is represented by some $f \in Z^{j i}$, then let 


$$
\begin{aligned}
{\left[\delta_{G / H}^{j i} f\right] } & \left(\hat{s}_{1}, \cdots, \hat{s}_{j+1}\right)\left(h_{1}, \cdots, h_{i}\right) \\
& =\hat{s}_{1}^{*}\left[f\left(\hat{s}_{2}, \cdots, s_{j+1}\right)\left(\hat{s}_{1}^{*} h_{1} \hat{s}_{1}^{*-1}, \cdots, \hat{s}_{1}^{*} h_{i} \hat{s}_{1}^{*-1}\right)\right] \\
& +\left[\sum_{k=1}^{j}(-1)^{k} f\left(\hat{s}_{1}, \cdots \hat{s}_{k} \hat{s}_{k+1}, \cdots, \hat{s}_{j+1}\right)+(-1)^{j+1} f\left(\hat{s}_{1}, \cdots \hat{s}_{j}\right)\right]\left(h_{1}, \cdots, h_{i}\right)
\end{aligned}
$$

where $\hat{s}_{1}^{*}$ is the coset representative of $\hat{s}_{1}$ chosen above. Since $\hat{s}_{1}^{*}$ is a Borel function of $s_{1}$ and $f$ is a Borel function of its $i+j$ variables, it is evident that $\delta_{\mathbf{G} / \mathbf{H}}^{\prime j i}$ is a Borel function of its $i+j+1$ variables. In virtue of the regularity, this map results in a homomorphism $\delta_{G / H}^{j i}$ of $\widetilde{C}^{j}\left(G / H, H^{i}(H, A)\right)$ into $\widetilde{C}^{j+1}\left(G / H, H^{i}(H, A)\right)$. It follows directly from $\mathrm{H}-\mathrm{S}$ that $\delta_{G / H}^{j i}$ is independent of the coset representatives used, that $C^{*}\left(G / H, H^{i}(H, A)\right)$ is a complex and that the homomorphism $r_{j}^{*}$ is a homomorphism of complexes of $E_{1}^{* i}$ (differentiations $d_{1}$ ) with $C^{*}\left(G / H, H^{i}(H, A)\right.$ ), so that

$$
r_{j}^{*}\left(d_{1} \alpha\right)=(-1)^{i} \delta_{G / H}^{j i}\left(r_{j-1}^{*} \alpha\right), \quad \alpha \in E_{1}^{j-1, i} .
$$

Thus we have the following result.

LEMMA 1.2. If $H^{i}(H, A)$ is regular in dimension $i$, then $r_{j}^{*}$ induces isomorphisms

$$
E_{2}^{j i} \simeq H^{j}\left(\tilde{C}^{*}\left(G / H, H^{i}(H, A)\right)\right.
$$

In case $i=0$, we recall that $E_{1}^{j 0} \simeq C^{j}\left(G / H, A^{H}\right)$. It is evident that $\delta_{G / H}^{j 0}$ is the usual differentiation on the complex $C^{*}\left(G / H, A^{H}\right)$ and thus $E_{2}^{j 0}$ is isomorphic to $H^{j}\left(G / H, A^{B}\right)$ where $A^{H}$ is viewed naturally as a $G / H$ module. Similarly, we see that $E_{2}^{0 i} \simeq H^{i}(H, A)^{G / H}$ for all $i$ (elements left fixed by the natural operation of $G / H$ on $\left.H^{i}(H, A)\right)$.

4. In this section we shall show how the difficulties described following Lemma 1.1 can be successfully surmounted in the case $i=1$.

Lemma 1.3. The complex $C^{*}(H, A)$ is regular in dimension one.

Proof. Since $A^{H}$ is a closed subgroup of $A$, we may choose a cross section $M$ of $A / A^{H}$ in $A$ which satisfies the conditions of Proposition 1.5. The map $\psi=\delta_{0} / M$ is then a one-to-one map of $M$ onto $B^{1}(H, A)\left(=B^{1}\right)$. The group $Z^{1}(H, A)\left(=Z^{1}\right)$ consists of continuous functions from $H$ to $A$ and is a separable metric space in the compact open topology. It is evident that $\psi$ is a Borel function from $M$ into $Z^{1}$ given the Borel structure deduced from its topology (since $\delta_{0}$ is a continuous function from $A$ to $Z^{1}$ ). By Souslin's theorem [15, p. 397] applied to $\psi$, we conclude that $\psi^{-1}$ is a Borel map from $B^{1}$ onto $M$. Finally, it is immediate that $\psi^{-1}=\phi_{1}$ satisfies the conditions of Definition 1.5. Q.E.D.

The remaining problem is to show that the functions of $E_{1}^{j 1}$ coincide with the 
Borel functions from $(G / H)^{j}$ to $H^{1}(H, A)\left(=H^{1}\right)$ for a suitable Borel structuer on the latter group. If the subgroup $B^{1}$ of $Z^{1}$ is closed (as it nearly always will be), then $H^{1}$ becomes a topological group, inheriting its topology frcm that of $Z^{1}$. The discussion leading to the proof of Lemma 1.2 shows that $G / H$ operates as a group of automorphisms of $H^{1}$. We shall now show that this operation is continuous. In order to do so, it is enough to show that the operation of $G$ on $Z^{1}$ from which it is derived is continuous.

If this is not the case, then there is a sequence $s_{n} \rightarrow s_{0}$ in $G$, a sequence $f_{n} \rightarrow f_{0}$ in $Z^{1}$, a compact set $C$ in $H$ with a sequence $h_{n}$ in $C$ converging to $h_{0}$, and a neighborhood $U$ of the identity in $A$ so that $s_{n} \cdot f_{n}\left(s_{n} h_{n} s_{n}^{-1}\right)-s_{0} \cdot f_{0}\left(s_{0} h_{0} s_{0}^{-1}\right) \notin U$. Since all the $f_{n}$ are continuous and since $s_{n} h_{n} s_{n}^{-1} \rightarrow s_{0} h_{0} s_{0}^{-1}$ while remaining within a compact set of $H$, it follows from Theorem 5, p. 223 of [14] that $f_{n}\left(s_{n} h_{n} s_{n}^{-1}\right) \rightarrow f_{0}\left(s_{0} h_{0} s_{0}^{-1}\right)$. But then since $G$ operates continuously on $A$,

$$
s_{n} f_{n}\left(s_{n} h_{n} s_{n}^{-1}\right) \rightarrow s_{0} f_{n}\left(s_{n} h_{n} s_{n}^{-1}\right),
$$

and this is a contradiction.

Therefore $H^{1}$ given the Borel structure generated by the topology on $H^{1}$ is a Borel $G / H$-module. (If $H^{1}$ is locally compact as it will usually be, it is also a topological $G / H$-module.) In any case the cochain complex $C^{*}\left(G / H, H^{1}(H, A)\right)$ has sense. Its relation to $\tilde{C}^{*}\left(G / H, H^{1}(H, A)\right)$ is expressed in the following lemma.

LEMMA 1.4. If either (a) $Z^{1}$ is locally compact and $B^{1}$ is closed or (b) $B^{1}=0$, then $\widetilde{C}^{*}\left(G / H, H^{1}(H, A)\right)=C^{*}\left(G / H, H^{1}(H, A)\right)$.

Proof. Let $F$ be an element of $\widetilde{C}^{j}\left(G / H, H^{1}(H, A)\right)$ and let $f$ be a representative of the class of functions $F$. Then $f$ is a $j$ dimensional cochain of $G / H$ taking values in the group $Z^{1}$, subject to the condition that the function $\tilde{f}, \hat{f}\left(h, \hat{s}_{1}, \cdots, \hat{s}_{j}\right)$ $=f\left(\hat{s}_{1}, \cdots, \hat{s}_{j}\right)(h)$ is a Borel function from $(G / H)^{j} \times H$ to $A$. We contend moreover that $f$ is a Borel function from $(G / H)^{j}$ to the topological group $Z^{1}$. For this, it is sufficient to show that $f^{-1}(K)$ is a Borel set in $(G / H)^{j}$ when $K$ is a set in $Z^{1}$ of the form, $\left\{\chi: \chi(h)-\chi_{0}(h) \in U\right.$ for all $\left.h \in C\right\}$ where $U$ is a closed neighborhood of the identity in $A, C$ a compact set in $H$ and $\chi_{0} \in Z^{1}$. If $h_{i}$ is a countable dense set in $C$, then

$$
f^{-1}(K)=\bigcap_{i=1}^{\infty}\left\{\left(\hat{s}_{1}, \cdots, \hat{s}_{j}\right): \hat{f}\left(h_{i}, \hat{s}_{1}, \cdots, \hat{s}_{j}\right)-\chi_{0}\left(h_{i}\right) \in U\right\}
$$

since $f$ and $\chi_{0}$ are continuous with respect to the variable $h$, and since $U$ is closed. Now as $\tilde{f}$ is a Borel function with respect to all of its variables, $f^{-1}(K)$ is seen to be a Borel set. If we apply to $f$ the continuous projection map of $Z^{1}$ onto $H^{1}$, there results a Borel $j$-cochain $\psi(F)$ of $G / H$ in the $G / H$ module $H^{1}$. That $\psi^{j}(F)$ depends only on $F$ as indicated and not on $f$ is self-evident. Finally it is also clear in virtue of Lemma 1.3 and the discussion following Definition 1.5 that $\psi^{j}$ is an injective homomorphism into the group of Borel $j$-cochains $C^{j}\left(G / H, H^{1}(H, A)\right)$. 
Conversely let $G$ be an element of this last group. Under the conditions enumerated above it is possible to make a choice of coset representatives $\hat{\chi} \rightarrow \hat{\chi}^{*}$ in $Z^{1}$ of cosets of $B^{1}$ so that $\hat{\chi} \rightarrow \hat{\chi}^{*}$ is a Borel function from $H^{1}$ to $Z^{1}$. Consequently the function $g$, defined by $g\left(\hat{s}_{1}, \cdots, \hat{s}_{j}\right)=G\left(\hat{s}_{1}, \cdots, \hat{s}_{j}\right)^{*}$, is a Borel function from $(G / H)_{j}$ to $Z^{1}$. As above, corresponding to $g$, we have a function $\tilde{g}$ of $j+1$ variables $\tilde{g}\left(h, \hat{s}_{1}, \cdots, \hat{s}_{j}\right)=g\left(\hat{s}_{1}, \cdots, \hat{s}_{j}\right)(h)$. If the argument $h$ in $H$ is fixed, it is clear from the nature of the compact open topology of $Z^{1}$ that $\tilde{g}$ is a Borel function with respect to the $j$ arguments in $G / H$. On the other hand if the $j$ arguments in $G / H$ are fixed, $\tilde{g}$ as a function of $h$ alone is continuous since it belongs to $Z^{\mathbf{1}}$. In virtue of Théorème 2, p. 285 of [15], $\tilde{g}$ is a Borel function of its $j+1$ variables. Thus $g$ represents a class $F$ of $\tilde{C}^{j}\left(G / H, H^{1}(H, A)\right)$ by definition of this group. Since it is clear that $\psi^{j}(F)=G$, we see that $\psi^{j}$ is an isomorphism. Thus the elements of $\tilde{C}^{j}\left(G / H, H^{1}(H, A)\right)$ viewed as $j$-cochains of $G / H$ in $H^{1}(H, A)$ coincide exactly with the class of Borel cochains of $G / H$ in $H^{1}(H, A)$. Q.E.D.

Moreover, the map $\psi^{j}$ taken together constitute an isomorphism of the two complexes $\widetilde{C}^{*}\left(G / H, H^{1}(H, A)\right)$ and $C^{*}\left(G / H, H^{1}(H, A)\right)$.

THEOREM 1.1. If either (a) $Z^{1}(H, A)$ is locally compact and $B^{1}(H, A)$ is closed or (b) $B^{1}(H, A)=0$, then $r_{j}^{*}$ and $\psi^{j}$ induce isomorphisms

$$
\begin{aligned}
& E_{1}^{j 1} \approx C^{j}\left(G / H, H^{1}(H, A)\right), \\
& E_{2}^{j 1} \approx H^{j}\left(G / H, H^{1}(H, A)\right),
\end{aligned}
$$

where $H^{1}(H, A)$ is given the Borel structure described above.

Proof. Lemmas 1.2, 1.3, and 1.4.

REMARKS. (1) The results through Lemma 1.3 are valid when suitably modified for the bounded theory. The obvious analogue of Theorem 1.1 for the bounded theory fails to be true in general. (For instance, let $G=H \oplus G / H=R \oplus G / H$, $A=A_{1} \oplus A_{2}=T \oplus R$ with $G$ operating trivially; then

$\left.\bar{E}_{2}^{j 1} \simeq H^{j}\left(G / H, H^{1}(R, T)\right) \oplus \bar{H}^{j}\left(G / H, H^{1}(R, R)\right)=H^{j}(G / H, R) \oplus \bar{H}^{j}(G / H, R).\right)$

This is the principal reason for not using the bounded theory throughout.

(2) If $G / H$ is discrete and if $H^{i}(H, A)$ is countable, then the term $E_{2}^{j i}$ of the spectral sequence of the extension is isomorphic to $H^{j}\left(G / H, H^{i}(H, A)\right)$ where $H^{i}(H, A)$ is given the discrete topology. We omit the trivial verification of this fact.

5. The spectral sequence of a group extension $G$ supplies a filtering on $H^{n}(G, A)=K_{0}^{n} \supseteq K_{1}^{n} \supseteq \cdots \supseteq K_{n}^{n} \supseteq 0$. (We shall delete the superscripts if there is no confusion about the dimension.) As in $\mathrm{H}-\mathrm{S}$, the composed map $H^{j}\left(G / H, A^{H}\right) \simeq E_{2}^{j 0} \rightarrow E_{\infty}^{j 0} \simeq K_{j}^{j} \subseteq H^{j}(G, A)$ is inflation from $G / H$ to $G$ and the composed map $H^{i}(G, A) \rightarrow K_{0}^{i} / K_{1}^{i} \simeq E_{\infty}^{0 i} \rightarrow E_{2}^{0 i} \simeq H^{i}(H, A)^{G / H}$ is restriction from $G$ to $H$. From p. 82 of [7], we have an exact sequence (the inflation-restriction sequence) 


$$
\begin{aligned}
0 \longrightarrow H^{1}\left(G / H, A^{H}\right) & \stackrel{\inf }{\longrightarrow} H^{1}(G, A) \stackrel{\text { res }}{\longrightarrow} H^{1}(H, A)^{G / H} \\
\stackrel{d_{2}}{\longrightarrow} H^{2}\left(G / H, A^{H}\right) & \stackrel{\operatorname{nf}}{\longrightarrow} K_{2} \subset K_{1} \subset K_{0}=H^{2}(G, A) .
\end{aligned}
$$

For convenience, we shall call $-d_{2}$ the transgression map, tg. It is important to have at least in a special case an explicit representation of $\mathrm{tg}$ in terms of cocycles. In fact if $H$ is abelian and $A^{H}=A$, then $(\hat{s}, \hat{t}) \rightarrow \chi(f(\hat{s}, \hat{t}))(\hat{s}, \hat{t} \in G / H)$ is a cocycle representative of $\operatorname{tg}(\chi)$ where $f$ is a 2-cocycle of $G / H$ in $H$ which defines the extension $G$ and $\chi \in H^{1}(H, A)^{G / H}$.

In applying these results the following fact concerning the cohomology of finite groups will be useful. We state it here for lack of a better place.

Proposition 1.8. Let $G$ be a finite group and $A$ a G-module. Then if $A$ is finite generated, $H^{r}(G, A), r \geqq 1$ are finite groups. If $A$ is countable, then so are the groups $H^{r}(G, A), r \geqq 0$.

\section{Chapter II}

1. In this chapter we commence our study of the global structure of the cohomology groups defined in the first chapter. This chapter is devoted to the case in which the group $G$ is compact, while later chapters will examine the same problems for a wider class of locally compact groups $G$. The first theorem (Theorem A) is fundamental for much of our work in this and later chapters. The content of this theorem is not new; it has been stated and proved in various forms by several authors $[2 ; 3]$. For the sake of completeness we state and prove it here in terms of the present terminology and in the precise form in which we shall need it.

We consider the exact sequence of topological groups

$$
0 \rightarrow Z \rightarrow R \stackrel{\pi}{\rightarrow} T \rightarrow 0 ;
$$

this sequence becomes a $G$-sequence for any locally compact group $G$ by letting $G$ operate trivially. The fundamental theorem is then the following.

THEOREM A. Let $G$ be a connected, simply-connected Lie group; then the map $H^{2}(G, R) \rightarrow^{\pi^{*}} H^{2}(G, T)$ is bijective.

Proof. We first show that $\pi^{*}$ is surjective. Let $a \in H^{2}(G, T)$ and let $\alpha^{\prime}$ be a a cocycle representative. We may then find a projective $\alpha^{\prime}$ representation $s \rightarrow L_{s}$ of $G$ (the left regular $\alpha^{\prime}$ representation, p. 274 of [19], for instance). If $s \rightarrow \tilde{L}_{s}^{\prime}$ denotes the corresponding homomorphism into the projective unitary group, $\tilde{L}^{\prime}$ is strongly continuous on $G$. Thus by a lemma of Bargmann [2, p. 6] we may find a neighborhood $U$ of the identity in $G$ and a projective representation $L$ of $G$ so that

(1) $\tilde{L}^{\prime}=\tilde{L}$ and thus the cocycle $\alpha$ attached to $L$ is equivalent to $\alpha^{\prime}$.

(2) $\alpha$ and $L$ are continuous functions on $U \times U$ and $U$ respectively. 
Now if we adopt Bargmann's terminology, $\alpha \uparrow U \times U$ is a local multiplier on $G$, and $U$ may be taken so that there is a local exponential multiplier $\beta^{\prime}$ on $U \times U$ corresponding to $\alpha \uparrow U \times U$. Moreover $\alpha$ and $\beta^{\prime}$ on $U \times U$ may be taken to be $C^{\infty}$ functions. Now Theorem 5.1 of [2] may be applied; $\beta^{\prime}$ may be extended to a continuous (in fact $C^{\infty}$ ) global exponential multiplier on $G$, let us say $\beta^{\prime \prime}$. Let $b \in H^{2}(G, R)$ be the cohomology class of $\beta^{\prime \prime}$ and let $\beta$ be image of $\beta^{\prime \prime}$ under $\pi$. The cocycle $\alpha-\beta$ is identically zero on $U \times U$ and if $s \rightarrow M_{s}$ is a projective representation corresponding to $\alpha-\beta, M$ is continuous on $U$ (see Appendix) and is a local homomorphism. As $G$ is connected and simply connected, $M \uparrow U$ may be extended to a representation of $G$, let us say $M^{\prime}$. Passing to the projective unitary group, we see that $\tilde{M}_{s}=\tilde{M}_{s}^{\prime}$ for $s \in U$ and hence for all $s \in G$. It follows then that the cocycle attached to $M$ is equivalent to the one attached to $M^{\prime}$; that is to say, $\alpha-\beta \sim 0$. It is immediate that $\pi^{*}(b) \simeq a$ and that $\pi^{*}$ is surjective.

To see that $\pi^{*}$ is injective, we could argue in the vein of the above; however, it is simpler to remark first that $H^{2}(G, Z)$ is isomorphic to $\operatorname{Ext}(G, Z)$ by Chapter $I$. As $G$ is connected and simply connected, we see easily that $H^{2}(G, Z)=0$. That $\pi^{*}$ is injective then follows from the exactness of the sequence.

$$
\rightarrow H^{2}(G, Z) \rightarrow H^{2}(G, R) \rightarrow H^{2}(G, T) \rightarrow .
$$

We have several remarks and complements to add to this theorem.

(1) Under the same hypotheses it is a trivial generalization that the natural map $H^{2}\left(G, R^{n}\right) \rightarrow H^{2}\left(G, T^{n}\right)$ is an isomorphism ( $G$ of course operates trivially on the two groups $R^{n}$ and $\left.T^{n}\right)$.

(2) If $G$ is as in Theorem A and operates on $R^{n}$, then $H^{2}\left(G, R^{n}\right) \approx H^{2}\left(g, R^{n}\right)$, where $g$ is the (real) Lie algebra of $G$ and operates on $R^{n}$ which may be identified to the Lie algebra of the vector group $R^{n} ; H^{2}\left(g, R^{n}\right)$ is formed in the sense of [4]. Bargmann [2, pp. $28 \mathrm{ff}$.] proves essentially this for $n=1$ and trivial operation of $G$. The same techniques produce a proof of the general statement above. The existence of such an isomorphism is rendered all the more evident when the respective cohomology groups are viewed in terms of extensions.

A similar argument establishes an analogous isomorphism $H^{1}\left(G, R^{n}\right) \simeq H^{1}\left(g, R^{n}\right)$

2. In this section the circle group $T$ is viewed as a trivial $G$ module where $G$ is restricted to a compact group. We first observe that $H^{2}(G, T)$ is necessarily a torsion group. Indeed if $a \in H^{2}(G, T)$, and if $\alpha$ is a cocycle representative, we may find an irreducible projective $\alpha$ representation $L$ of $G$. Such representations correspond uniquely to certain irreducible representations of the group extension $G^{\alpha}$ of $G$ by $T\left[19\right.$, p. 269]. The group $G^{\alpha}$ is compact since $G$ and $T$ are, and it follows that $L$ is finite dimensional. The function $\gamma(s)=\operatorname{det} L_{s}$ is a Borel function on $G$ to $T$, and it follows from the relation $L_{s} L_{t}=\alpha(s, t) L_{s t}$ that $\alpha(s, t)^{n}=\gamma(s) \cdot \gamma(t) / \gamma(s t)$ where $n=\operatorname{dim} L$. This relation implies $a^{n}=1$. The essence of this argument is of course not new (cf. [25, p. 183; 2, p. 10]). We shall 
now proceed toward the first main theorem by way of some preliminary results. The content of the first of these (Proposition 2.1) has been given previously $[3 ; 24]$.

Proposition 2.1. Let $G$ be a compact connected Lie group; then there is a canonical isomorphism: $H^{2}(G, T) \approx\left(t\left(\pi_{1}(G)\right)\right)^{\wedge}$.

Proof. If $\widetilde{G}$ is the universal covering group of $G, \pi_{1}(G)$ is isomorphic to a central subgroup of $\tilde{G}$ and $G \approx \tilde{G} / \pi_{1}(G)$. The restriction inflation sequence corresponding to $\pi_{1}(G)$ reads as follows:

$$
\cdots \rightarrow H^{1}(\tilde{G}, T) \stackrel{\text { res }}{\longrightarrow} H^{1}\left(\pi_{1}(G), T\right) \stackrel{\text { inf }}{\longrightarrow} H^{2}(G, T) \rightarrow H^{2}(\tilde{G}, T) .
$$

By Theorem $\mathrm{A}, H^{2}(\tilde{G}, T)$ is isomorphic to a vector group, and by the result above $H^{2}(\widetilde{G}, T)$ is a torsion group; thus, the map inf must be the trivial map, and we have

$$
H^{2}(G, T) \simeq H^{1}\left(\pi_{1}(G), T\right) / \text { range(res) } .
$$

To complete the proof it is enough now to show that the group $A=\operatorname{range(res)}$ coincides with the group $B=\left\{\chi \in \pi_{1}(G): \chi\left(t\left(\pi_{1}(G)\right)\right)=0\right\}$. The group $\tilde{G} /[\overline{\tilde{G}, \tilde{G}}]$ is known to be a vector grcup and so its dual $H^{1}(\widetilde{G}, T)$ is also a vector group. Moreover the map res is a continuous map into $\left(\pi_{1}(G)\right)^{\wedge}$. Therefore $A$ is contained in the connected component of $\left(\pi_{1}(G)\right)^{\wedge}$ but this latter is none other than the group $B$; thus $A \subseteq B$. The group $B / A$ is isomorphic to a subgroup of the torsion group $H^{2}(G, T)$ and is thus a torsion group. On the other hand since $\pi_{1}(G)$ is finitely generated, $B$ is a Lie group, and the remarks above show that $A$ is a Lie subgroup of $B$. However it is evident that such a group can never be a torsion group unless $A=B$. Q.E.D.

An immediate corollary of Proposition 2.1 is that $H^{2}(G, T)$ is a finite group. We shall now extend this last statement to a wider class of groups.

Proposition 2.2. If $G$ is a compact Lie group, then $H^{2}(G, T)$ is a finite group.

Proof. Denote by $G_{0}$ the connected component of $G$. Since $G_{0}$ is a normal subgroup there is a spectral sequence $\left\{E_{r}^{j i}\right\}$ abutting to $H^{*}(G, T)$. In order to show that $H^{2}(G, T)$ is finite, it is enough to show that the graded group $\sum_{i+j=2} \bigoplus E_{\infty}^{j i}$ attached to the filtered group $H^{2}(G, T)$ is finite, and in turn it suffices to show that the groups $E_{2}^{j i}$ for $i+j=2$ are finite. By Chapter I, $E_{2}^{02} \simeq H^{2}\left(G_{0}, T\right)$, which is a finite group by the preceding proposition. Also by Theorem 1.1 we know that $E_{2}^{11} \simeq H^{1}\left(G / G_{0}, H^{1}\left(G_{0}, T\right)\right)$; now $H^{1}\left(G_{0}, T\right)$ is finitely generated and $G / G_{0}$ is finite so that by Propostion $1.8 E_{2}^{\mathbf{1 1}}$ is finite. Finally $E_{2}^{20} \simeq H^{2}\left(G / G_{0}, T\right)$ and this final group is isomorphic to $H^{3}\left(G / G_{0}, Z\right)$ by "dimension shifting." We conclude then, again by Proposition 1.8, that $E_{2}^{20}$ is finite, and our result follows. 
If $G$ is an arbitrary compact group, it is known that $G$ may be represented as the inverse limit of a family of compact Lie groups: $G=\lim p{ }_{\alpha} G_{\alpha}$. The following theorem provides a corresponding relation for the two dimensional cohomology groups.

THEOREM 2.1. If $G=\lim p{ }_{\alpha} G_{\alpha}$ where the $G_{\alpha}$ are compact groups, then

$$
H^{2}(G, T) \simeq \lim d \cdot{ }_{\alpha} H^{2}\left(G_{\alpha}, T\right) \text {. }
$$

Proof. The natural inflation maps $H^{2}\left(G_{\alpha}, T\right) \rightarrow H^{2}\left(G_{\beta}, T\right)$ for $\alpha \leqq \beta$ define together with the groups $H^{2}\left(G_{\alpha}, T\right)$ an inductive system. Let $K$ denote the limit group. Further there are inflation maps $H^{2}\left(G_{\alpha}, T\right) \rightarrow H^{2}(G, T)$ for all $\alpha$; and granting the trivial fact of their compatibility, we deduce from the universal property of $K$ a homomorphism $\phi: K \rightarrow H^{2}(G, T)$.

(a) $\phi$ is injective. Let $\tilde{a} \in K$; then $\tilde{a}$ may be represented by an $a \in H^{2}\left(G_{\alpha}, T\right)$ for some $\alpha$. Then if $\phi(\tilde{a})=0$, the element $a$ upon inflation to $G$ is trivial. In virtue of the exactness of the following sequence $H^{1}\left(H_{\alpha}, T\right)^{G_{\alpha}} \stackrel{\text { tg }}{\rightarrow} H^{2}\left(G_{\alpha}, T\right)$ $\rightarrow H^{2}(G, T)\left(H_{\alpha}\right.$ denotes the kernel of the projection $\left.G \rightarrow G_{\alpha}\right)$, there is an element $\chi \in H^{1}\left(H_{\alpha}, T\right)^{G_{\alpha}}$ so that $\operatorname{tg} \chi=a$. Now by a well-known theorem of von Neumann, $H_{\alpha} /(\operatorname{ker} \chi)$ is a Lie group. The groups $H_{\beta}$ for $\beta \geqq \alpha$ are arbitrarily small subgroups of $H_{\alpha}$, and therefore the groups $\left(H_{\beta} \cdot \operatorname{ker} \chi\right) / \operatorname{ker} \chi \downarrow(e)$. Since the latter is a Lie group, we see that $H_{\beta} \subseteq \operatorname{ker} \chi$ for some $\beta \geqq \alpha$. If $\operatorname{tg}_{\beta}$ denotes the map of $H^{1}\left(H_{\beta}, T\right)^{G_{\rho}}$ into $H^{2}(G, T), \chi^{\prime}$ the restriction of $\chi$ to $H_{\beta}$, and $a^{\prime}$ the inflation of $a$ to $G_{\beta}$, it follows easily that $a^{\prime}=\operatorname{tg}_{\beta} \chi^{\prime}=0$ since $\chi^{\prime}=0$. The fact that the original element $a$ inflates to zero in some $G_{\beta}$ for $\beta \geqq \alpha$ is merely the fact that $\tilde{a}=0$ in $K$. Thus $\phi$ is injective.

(b) $\phi$ is surjective. If $a \in H^{2}(G, T)$ is given, we must then show that $a$ arises by inflation from some $G_{\beta}$. Let $a$ be a cocycle representative; we may find as before a finite dimensional projective $a$ representation $L$. If $\tilde{L}$ denotes the corresponding homomorphism into the projective unitary group (a Lie group), and $K$ the kernel of $\tilde{L}$, then, as we have argued in part (a), using the theorem of von Neumann there is some index $\beta$ for which $H_{\beta} \subseteq K$. Then $\tilde{L}$ can be viewed as a homomorphism of $G / H_{\beta}=G_{\beta}$ into the projective unitary group, and then we may choose (cf. [19, p. 271]) a corresponding projective representation $M^{\prime}$ of $G_{\beta}$. Finally let $b^{\prime}$ be the 2-cocycle attached to $M^{\prime}$, and $b$ the class of $b^{\prime}$. Upon inflation from $G_{\beta}$ to $G, M^{\prime}$ becomes a projective representation $M$ of $G$ with associated cocycle $b$, where $b(s, t)=b^{\prime}(\hat{s}, \hat{t})$ and $M_{s}=M_{s}^{\prime}(s, t \in G, \hat{s}, \hat{t}$, their images in $G_{\beta}$ ). The class of $b$ is $\inf (b)$. Now $\tilde{M}_{s}=\tilde{M}^{\prime}{ }_{s}=\tilde{L}_{s} \mathrm{~J}$ by construction. Since for any projective $\gamma$ representation $N$ of $G$ the homomorphism $N$ of $G$ into the projective unitary group determines the class of $\gamma$ uniquely, we deduce from the foregoing that $b \sim a$; that is, $a=\inf (b)$. Q.E.D.

We have the following corollary of these results.

CoROllary 1. If $G$ is a compact group, $H^{2}(G, T)$ is a countable torsion group. 
Proof. Since $G$ is separable we may write $G$ as the inverse limit of a sequence $G_{i}$ of compact Lie groups. The result follows from Proposition 2.1 and Theorem 2.1.

This corollary does supply some insight into the cohomology group in question; it is also as far as we can go for it is fairly easy to see by direct construction that any countable torsion group can be obtained as $H^{2}(G, T)$ by a suitable choice of $G$. This we shall do later. This corollary also bears on the problem of assigning a topology to the group of extensions $H^{2}(G, T)$; for if this group has a locally compact topology, this topology must be discrete in virtue of the countability. This observation is grantedly ad hoc; however, it will be subsumed in a general theory later.

Theorem 2.1 may be used to prove an analogue of itself where $T$ is replaced by a finite group. Any finite group $A$ is a $G$-module by giving $G$ trivial action. Then $A$ is a direct sum of cyclic groups as $G$-module and it suffices to prove the assertions of Theorem 2.1 for a cyclic group $J_{n}$ of order $n$. The group $J_{n}$ is a subgroup of $T$ and the following sequence is exact

$$
0 \rightarrow J_{n} \stackrel{i}{\rightarrow} T \stackrel{j}{\rightarrow} T \rightarrow 0
$$

where $j$ is multiplication by $n$. We have then an exact sequence of cohomology

$\left(^{*}\right) \rightarrow H^{1}(G, T) \stackrel{j}{\rightarrow} H^{1}(G, T) \stackrel{\delta}{\rightarrow} H^{2}\left(G, J_{n}\right) \stackrel{i}{\rightarrow} H^{2}(G, T) \stackrel{j}{\rightarrow} H^{2}(G, T) \rightarrow$.

Now $J_{n}$ is of exponent $n$ so that $H^{2}\left(G, J_{n}\right)$ is also. If $G$ is a Lie group, $H^{1}(G, T)$ is finitely generated so that $\delta\left(H^{1}(G, T)\right)$, being a torsion group, is finite. Since $H^{2}(G, T)$ is finite by Theorem $2.1, H^{2}\left(G, J_{n}\right)$ is finite. Finally if $G=\lim p{ }_{a} G_{a}$, we may form sequences $\left({ }^{*}\right)$ with $G_{\alpha}$ in place of $G$. We take the inductive limit of the five term exact sequences over the index set to obtain an exact sequence $\left({ }^{* *}\right)$. In each term in $\left(^{*}\right), H^{i}(G, A)$, is replaced by $\lim d{ }_{\alpha} H^{i}\left(G_{\alpha}, A\right)$. We further have a map of the five term sequence $\left({ }^{* *}\right)$ into the five term sequence $\left({ }^{*}\right)$; that is, five maps $\left\{\phi_{i}\right\}_{i=1}^{5}$ reading from left to right. Now $\phi_{1}, \phi_{2}, \phi_{4}, \phi_{5}$ are known to be bijective; by the five lemma, $\phi_{3}$ is bijective; that is, the natural map $\phi_{3}: \lim d{ }_{\alpha} H^{2}\left(G_{\alpha}, J_{n}\right)$ into $H^{2}\left(G, J_{n}\right)$ is bijective.

CoRollary 2. If $G$ is compact, Theorem 1.2 remains valid if $T$ is replaced by any finite group $A$ with $G$ acting trivially.

3. In this section we shall extend our previous results to the case when a compact group $G$ operates in any continuous fashion on a finite dimensional torus or a finite group $A$. Since $\operatorname{Aut}(A)$ is discrete (cf. [12, p. 509]), it follows that $G / G_{0}$ is finite where we use $G_{0}$ to denote the kernel of the homomorphism of $G$ into $\operatorname{Aut}(A)$. The result for dimension one below will be used in connection with the spectral sequence of a group extension. The result for dimension three will be used later also. 
THeOREM 2.2. (1) Let $G$ and $A$ be as above; then $H^{1}(G, A)$ is countable and the compact open topology on $B^{1}(G, A)$ induces on $H^{1}(G, A)$ the discrete topology.

(2) If $G$ is a Lie group, then $H^{2}(G, A)$ is finite.

(3) If $G=\lim p \cdot{ }_{\alpha} G_{\alpha}$, and if $L^{r}=\lim d \cdot{ }_{\alpha} H^{r}\left(G, A^{H_{\alpha}}\right)$ where $G_{\alpha}=G / H_{\alpha}$, then the natural map of $L^{r}$ into $H^{r}(G, A)$ is bijective for $r=1,2$ and injective for $r=3$.

Proof. We omit the simple proof of part (1). For part (2), we consider the spectral sequence $E_{r}^{i j}$ which abuts to $H^{*}(G, A)$. Now $E_{2}^{02} \simeq H^{2}\left(G_{0}, A\right)^{G / G_{0}}$ and this is finite since $G_{0}$ operates trivially and we may use the results of the last section. The group $H^{1}\left(G_{0}, A\right)$ is discrete in the compact open topology and finitely generated since $G_{0}$ is a Lie group. By Theorem 1.1 and Proposition 1.8 we deduce that $E_{2}^{11}$ is finite. Finally $E_{2}^{20} \simeq H^{2}\left(G, G_{0}, A\right)$ is easily seen to be finite by the cohomology of finite groups. Now since the terms of total degree two in $E_{2}$ are finite, it follows at once that $H^{2}(G, A)$ is finite.

We now pass to part (3). Since $G_{0}$ is open we may assume that $H_{\beta} \subset G_{0}$ for all $\beta$, since this does hold for a cofinal set of indices. It is clear that we do have maps $\phi^{r}$ from $L^{r}$ to $H^{r}(G, A)$. It is trivial that $\phi^{1}$ is bijective and the argument of Theorem 2.1 shows at once that $\phi^{2}$ is injective. To prove that $\phi^{2}$ is surjective, let $a \in H^{2}(G, A)$ and let $a^{\prime}$ be its restriction to $G_{0}$. By the previous section, we can find a net $a_{\alpha}^{\prime} \in H^{2}\left(G_{0} / H_{\alpha}, A\right)$ with $\phi^{2}\left(\lim a_{\alpha}^{\prime}\right)=a^{\prime}$. Since $G / G_{0}$ is finite, its orbit on $a_{\alpha}^{\prime}$ is finite and we may therefore suppose that $a_{\alpha}$ is invariant under $G / G_{0}$ for large $\alpha$. We want to prove that $a_{\alpha}^{\prime}$ is the restriction to $G_{0} / H_{\alpha}$ of an element $a_{\alpha}^{\prime \prime}$ of $H^{2}\left(G_{\alpha}, A\right)$ for large $\alpha$.

We introduce spectral sequences ${ }^{\alpha} E_{r}^{i j}$ abutting to $H^{*}\left(G_{a}, A\right)$ defined by the subgroup $G_{0} / H_{\alpha}$ of $G / H_{\alpha}=G_{\alpha}$ and a spectral sequence $E_{r}^{i j}$ abutting to $H^{*}(G, A)$ defined by $G_{0}$ which we used above. It is evident that we have a family of compatible maps ${ }^{\alpha} \psi_{r}^{i j}$ of ${ }^{\alpha} E_{r}^{i j}$ into $E_{r}^{i j}$ which commute with all the differentials of the spectral sequences. We need the following facts.

LEMMA 2.1. (1) $\lim d_{\alpha_{\alpha}}{ }^{\alpha} E_{2}^{i 1} \simeq E_{2}^{i 1}$ for $i=1,2$.

(2) For large $\alpha,{ }^{\alpha} \psi_{3}^{30}$ is an isomorphism of ${ }^{\alpha} E_{3}^{30}$ with $E_{3}^{30}$.

Proof. Since $G / G_{0}$ is finite and $H^{1}\left(G_{0} / H_{\alpha}, A\right)$ is countable, we deduce from the second remark after Theorem 1.1 that ${ }^{\alpha} E_{2}^{i 1} \simeq H^{i}\left(G / G_{0}, H^{1}\left(G_{0 \alpha} / H, A\right)\right)$ for all $i$, and similarly for $E_{2}^{i 1}$. But now $H^{1}\left(G_{0}, A\right) \simeq \lim d_{\alpha} H^{1}\left(G_{0} / H_{\alpha}, A\right)$ by the present theorem in dimension one, and since the cohomology of finite groups commutes with direct limits, the first part of the lemma is proved.

For the second part, ${ }^{\alpha} E_{2}^{30}=E_{2}^{30}=H^{3}\left(G / G_{0}, A\right)$ and ${ }^{\alpha} \psi_{2}^{30}$ is the identity map. It is easy to see that this is a finite group. But ${ }^{\alpha} E_{3}^{30}={ }^{\alpha} E_{2}^{30} /{ }^{\alpha} d_{2}^{11}\left({ }^{\alpha} E_{2}^{11}\right)$, and since $E_{2}^{11} \simeq \lim d_{\alpha}{ }_{\alpha}^{\alpha} E_{2}^{11}$ by part (1) of the lemma, ${ }^{\alpha} d_{2}^{11}\left({ }^{\alpha} E_{2}^{11}\right)=d_{2}^{11}\left(E_{2}^{11}\right)$ for large $\alpha$ by reasons of finiteness, and the lemma follows.

Let us return to the net $a_{\alpha}^{\prime} \in H^{2}\left(G_{0} / H_{\alpha}, A\right)^{G / G_{0}}={ }^{\alpha} E_{2}^{02}$. Now by the lemma for 
$E_{2}^{21},{ }^{a} d_{2}^{02}\left(a_{\alpha}^{\prime}\right)$ converges to $d_{2}^{02}\left(a^{\prime}\right)$ since $a_{\alpha}^{\prime}$ converges to $a^{\prime}$, but $d_{2}^{02}\left(a^{\prime}\right)=0$ since $a^{\prime}$ is the restriction of $a$ to $G_{0}$. Hence ${ }^{\alpha} d_{2}^{02}\left(a_{\alpha}\right)=0$ forlarge $\alpha$. For these $\alpha, a_{\alpha}^{\prime}$ may be viewed as an element of ${ }^{\alpha} E_{3}^{02}$ and $a_{\alpha}^{\prime}$ is extensible to $G_{\alpha}$ if and only if ${ }^{a} d_{3}^{02}\left(a_{\alpha}^{\prime}\right)=0$. But ${ }^{\alpha} d_{3}^{02}\left(a_{\alpha}^{\prime}\right)$ converges to $d_{3}^{02}\left(a^{\prime}\right)=0$ by part (2) of the lemma, and hence $a_{\alpha}^{\prime}$ is extensible to $G_{\alpha}$ for large $\alpha$.

Let $a_{\alpha}^{\prime \prime}$ be a net of such extensions which we may assume is defined for all $\alpha$. Let $a^{\prime \prime}=\phi^{2}\left(\lim a_{\alpha}^{11}\right)$ be the image of the limit in $H^{2}(G, A)$. But then $a-a^{\prime \prime}$ restricts to zero on $G_{0}$ and in order to prove that $\phi^{2}$ is surjective, it suffices to prove that every element in $K_{1}$, the first term of the filtering on $H^{2}(G, A)$ is in the image of $\phi^{2}$.

Let $a$ be such an element; then $\hat{a}$, its image in $K_{1} / K_{2}=E_{\infty}^{11} \subset E_{2}^{11}\left(K_{2}\right.$ is the range of inflation from $G / G_{0}$ ), may be viewed as an element of $E_{2}^{11}$ with $d_{2}^{11}(\hat{a})=0$. By the lemma, we can find a net $\hat{a}_{\alpha} \in{ }^{\alpha} E_{2}^{11}$ converging to $\hat{a}$. But ${ }^{\alpha} d_{2}^{11}\left(a_{\alpha}\right)$ converges to 0 since ${ }^{\alpha} E_{2}^{30}=E_{2}^{30}$ as we have remarked in the proof of the lemma. Thus ${ }^{\alpha} d_{2}^{11}\left(\hat{a}_{\alpha}\right)=0$ for large $\alpha$. It follows that there is a net $\tilde{a}_{\alpha} \in H^{2}\left(G / H_{\alpha}, A\right)$ with the property that $\tilde{a}=\phi^{2}\left(\lim \tilde{a}_{\alpha}\right)$ and $\tilde{a}-a$ viewed as an element of $K_{1} / K_{2}$ is zero. Thus it suffices to prove that the range of $\phi^{2}$ contains $K_{2}$ but this is trivial since $H_{\alpha} \subset G_{0}$ for all $\alpha$.

We will sketch the proof of the fact that $\phi^{3}$ is injective since the technique involved is quite similar to the one used above. Let $f_{\beta \alpha}$ be the inflation mapping from $H^{3}\left(G_{\alpha}, A\right)$ to $H^{3}\left(G_{\beta}, A\right)$ for $\beta \geqq \alpha$, and $f_{\alpha}$ the inflation map from $G_{\alpha}$ to $G$.

e must show that if $f_{\alpha}(a)=0$ for some $a \in H^{3}\left(G_{\alpha}, A\right)$, then $f_{\beta \alpha}(a)=0$ for large $\beta$. We introduce spectral sequences ${ }^{\beta} F_{r}^{i j}$ abutting to $H^{*}\left(G_{\beta}, A\right)$ defined by the normal subgroup $H_{\alpha} / H_{\beta}$ of $G / H_{\beta}=G_{\beta}$ for $\beta \geqq \alpha$, and a spectral sequence $F^{i j}$ abutting to $H^{*}(G, A)$ defined by $H_{\alpha}$. We have compatible homomorphisms of ${ }^{\beta} F_{r}^{i j}$ into $F_{r}^{i j}$ and of ${ }^{\beta} F_{r}^{i j}$ into ${ }^{\gamma} F_{r}^{i j}$ for $\gamma \geqq \beta$.

One easily establishes two isomorphisms:

and

$$
\lim d{ }_{\beta}{ }_{\beta} F_{3}^{02} \simeq F_{3}^{02}
$$

$$
\lim d \cdot{ }_{\beta}{ }^{\beta} F_{3}^{30} \simeq F_{3}^{30} .
$$

Suppose that $f_{\alpha}(a)=0$ for some $a \in H^{3}\left(G_{\alpha}, A\right)=F_{2}^{30}={ }^{\beta} F_{2}^{30}$. Then if $a^{\prime}$ is the image of $a$ in $F_{3}^{30}$, we know that there is some $b \in F_{3}^{02}$ such that $d_{3}^{02}(b)=a^{\prime}$. We can find by the first isomorphism a net $b_{\beta} \in{ }^{\beta} F_{3}^{02}$ converging to $b$. If $a_{\beta}^{\prime}$ is the image of $a$ in ${ }^{\beta} F_{2}^{30}$, it follows from the second isomorphism that ${ }^{\beta} d_{3}^{02} b_{\beta}-a_{\beta}^{\prime}$ converges to $d_{3}^{02}(b)-a^{\prime}=0$. Hence $a_{\beta}^{\prime}={ }^{\beta} d_{3}^{02} b_{\beta}$ for large $\beta$ and this means precisely that $f_{\beta x}(a)=0$. This completes the proof.

Corollary. If $G$ and $A$ are as above, then $H^{2}(G, A)$ is a countable torsion group.

We now state our final result for compact groups $G$. The case of a discrete $G$ module will be used later. 
THEOREM 2.3. If $A$ is an abelian Lie group which is a G-kernel, then

(1) $H^{r}(G, A)$ is denumerable, and $H^{r}(G, A)=0$ if $A$ is a vector group for $r=1,2$.

(2) If $G=\lim p_{\alpha} G_{\alpha}$, with $G_{\alpha}=G / H_{\alpha}, \lim d_{\alpha} H^{r}\left(G_{\alpha}, A^{B_{\alpha}}\right) \simeq H^{r}(G, A), r=1,2$.

Proof. Suppose that $A$ is a vector group; then $\bar{H}^{r}(G, A)=0$ for all $r>0$ since the usual averaging process $([13$, p. 535], $[2$, p. 19], and others) can be used. If $a$ is a bounded $r$-cocycle,

$$
b\left(s_{1}, \cdots, s_{r-1}\right)=\int_{G} s^{-1} \cdot a\left(s, s_{1}, \cdots, s_{r-1}\right) d \mu(s)
$$

is a bounded $r-1$ cochain with $\delta b=a$ ( $\mu$ is normalized Haar measure on $G$ ). The second statement of (1) follows by Proposition 1.7. If now $A$ is a connected group, let $K$ be the maximal torus so that $A / K$ is a vector group; then

$$
0 \rightarrow K \rightarrow A \rightarrow A / K \rightarrow 0
$$

is an exact $G$ sequence. Writing out the exact sequence of cohomology for the bounded theory and making use of the fact that $\bar{H}^{r}(G, A)=0$, we deduce as usual that $\bar{H}^{r}(G, A) \simeq H^{r}(G, K)=H^{r}(G, K)$ for $r>1$. Together with Theorem 2.2, this proves the present theorem for connected groups for $r=2$. The case $r=1$ is easy. We note that $\bar{H}^{3}(G, A)=H^{3}(G, K)$, a fact we shall need presently.

Let $A$ be as in the theorem, $A^{\prime}$ lts connected component and $A^{\prime \prime}=A / A^{\prime}$. Then $A^{\prime \prime}$ is discrete. We may find a sequence $A_{i}$ of subgroups of $A, A_{i} \supset A^{\prime}$ such that $A=\bigcup A_{i}$ and $A_{i} / A^{\prime}$ is finitely generated. Since $G$ is compact, the orbit of any $\hat{a} \in A_{i} / A$ is finite. It follows that we may take each $A_{i}$ to be in addition, $G$ invariant.

LEMmA 2.2. With the above notation,

$$
\lim d_{i} H^{r}\left(G / H, A_{i}^{H}\right) \simeq H^{r}\left(G / H, A^{\mathrm{B}}\right) \text { for } r=1,2
$$

and any normal subgroup $H$ of $G$.

Proof. It suffices to prove the result for $\bar{H}^{r}(\cdot, \cdot)$. Now a bounded cochain with values in $A^{H}$ takes on only a finite number of values modulo $A^{\prime} \cap A^{H}$. Then it follows just as for finite groups that we have a limit relation even at the cochain level; i.e.,

$$
\lim d{ }_{i} C^{*}\left(G / H, A_{i}^{H}\right) \simeq \bar{C}^{*}\left(G / H, A^{H}\right)
$$

and we are done.

Now let us return to the theorem. Suppose the theorem is true for each $A_{i}$, in place of $A$. Then using Lemma 1.2 for $H=(e)$ and $H=H_{a}$, we see that the theorem is true of $A$ by a simple iterated limit theorem for direct limits. Thus we are reduced to the case when $A^{\prime \prime}$ is finitely generated as an abelian group. 
Let us assume for the moment that the theorem is true for discrete $G$-kernels. Consider the exact $G$-sequence

$$
0 \rightarrow A^{\prime} \rightarrow A \rightarrow A^{\prime \prime} \rightarrow 0
$$

where $A^{\prime}$ is the connected component of $A$ and $A^{\prime \prime}$ is discrete. Part (1) is known for $A^{\prime}$ and $A^{\prime \prime}$ and the result follows for $A$ by looking at the exact sequence of cohomology of ( $\left(^{*}\right)$. Part (2) for $r=1$ is easy and we pass to the case $r=2$. A portion of the exact sequence of $\left(^{*}\right)$ reads as follows:

$$
\rightarrow H^{1}\left(G, A^{\prime \prime}\right) \rightarrow H^{2}\left(G, A^{\prime}\right) \rightarrow H^{2}(G, A) \rightarrow H^{2}\left(G, A^{\prime \prime}\right) \rightarrow H^{3}(G, K)
$$

where $K$ is the maximal torus in $A^{\prime}$. We can use $H^{3}(G, K)$ as final term since we could write the exact sequence of $\left(^{*}\right)$ in the bounded theory which is identical with (**) except for the last term which would be $\bar{H}^{3}\left(G, A^{\prime}\right)$. We have remarked before that this group is isomorphic to $H^{3}(G, K)$. As $A^{\prime \prime}$ is finitely generated it is easy to see that $A^{H_{\alpha}}=A$ for all large $\alpha$, and in that case $\left(^{*}\right)$ may be viewed as an exact $G_{\alpha}$-sequence for these $\alpha$. Then we have a five term exact sequence $\left.{ }^{(* *}\right)_{\alpha}$ identical to $\left({ }^{* *}\right)$ but with $G$ replaced by $G_{\alpha}$. We may take the limit of the five term sequences $\left({ }^{* *}\right)_{\alpha}$ to obtain another five term exact sequence $\left({ }^{* * *}\right)$ with each term $H^{r}(G, B)$ of $\left(^{* *}\right)$ replaced by $\lim d^{*}{ }_{\alpha} H^{r}\left(G_{\alpha}, B\right)$. Moreover we have compatible mappings $\phi_{1}, \cdots, \phi_{5}$ reading from left to right of $\left({ }^{* *}\right)$ into $\left(^{* *}\right)$. We want to know that $\phi_{3}$ is bijective. We know that $\phi_{1}, \phi_{2}$ and $\phi_{4}$ are bijective and that $\phi_{5}$ is injective, using Theorem 2.2 and our assumptions. It then follows from the five lemma that $\phi_{3}$ is bijective.

We are now finally reduced to the case $r=2$ and $A$ a finitely generated discrete group. If $A$ is torsion free, $A=Z^{n}$ for some $n$, and we write an exact sequence of groups:

$$
0 \rightarrow Z^{n} \rightarrow R^{n} \rightarrow T^{n} \rightarrow 0 .
$$

It is clear that this becomes in a natural way a $G$-sequence. Since $H^{r}\left(G, R^{n}\right)=0$ for $r=1,2$, it follows that $H^{2}\left(G, Z^{n}\right) \simeq H^{1}\left(G, T^{n}\right)$ by "dimension shifting." Since $H_{\alpha}$ acts trivially for large $\alpha$, our assertions are established by virtue of Theorem 2.2. If $A$ is a torsion group it is finite and our result follows from Theorem 2.2. In general we write an exact sequence

$$
0 \rightarrow A^{\prime} \rightarrow A \rightarrow A^{\prime \prime} \rightarrow 0
$$

with $A$ finite and $A^{n}=Z^{n}$ and we can repeat verbatim the five term exact sequence argument used above. All of our assertions are now completely proved.

\section{APPENDIX}

In the course of Theorem A (Chapter II) we needed to know that if $a$, an element of $Z^{2}(G, T)$, was continuous on $U \times U$ where $U$ is an open neighborhood of the identity in $G$, then any projective unitary representation $M$ with attached 
cocycle $a$ is continuous on $U$. In fact, let $G^{a}$ be the extension of $G$ by $T$ [19] defined by $a$ and let $M^{\prime}$ be the unitary representation of $G^{a}$ corresponding to $M$. The group $G^{a}$ can be represented as a set by the product $T \times G$ and $M$ is the restriction to $e \times G$ of $M^{\prime}$. Since $M^{\prime}$ is continuous, it suffices to show that the topology of $G^{a}$ restricted to $T \times U$ is the product topology. This is easily verified, for let us denote by $\Sigma$ the family of subsets of $G^{a}$ of the form $W \times V$ where $W$ is an open neighborhood of the identity in $T$ and $V$ is an open neighborhood of the identity in $G$ contained in $U$. The sets are easily seen to satisfy the five conditions in $[23$, p. 55]. The family $\Sigma$ defines a topology of this abstract group $G^{a}$ so that it becomes a topological group. Using the continuity of $a$ on $U \times U$, we see that this topology is the product topology in $T \times U$. Thus this topology is locally compact and its Borel structure is easily seen to be exactly that of the product $T \times G$. It follows from $[1$, p. 25], that this topology must coincide with the original locally compact topology of $G^{a}$ defined by means of a measure theoretic construction $[19$, p. 270$]$. This completes the proof of the desired result.

\section{BIBLIOGRAPHY}

1. S. Banach, Théorie des opérations linéares, Monogn Mat. Tom. 1, Warsaw, 1932.

2. V. Bargmann, On unitary ray representations of continuous groups, Ann. of Math. (2) 59 (1954), 1-46.

3. M. L. Calabi, Sur les extensions des groupes topologiques, Ann. Mat. Pura Appl. (4) 32 (1951), 295-370.

4. C. Chevalley and S. Eilenberg, Cohomology theory of Lie groups and Lie algebras, Trans. Amer. Math. Soc. 63 (1948), 85-124.

5. S. Eilenberg and S. MacLane, Cohomology theory in abstract groups. I, Ann. of Math. (2) 48 (1947), 51-78.

6. - Cohomology theory in abstract groups. II, Ann. of Math. (2) 48 (1947), 326-341.

7. R. Godement, Théorie des faisceaux, Hermann, Paris, 1958.

8. A. M. Gleason, Spaces with a compact Lie group of transformations, Proc. Amer. Math. Soc. 1 (1950), 35-43.

9. P. R. Halmos, Measure theory, Van Nostrand, New York, 1950.

10. G. Hochschild, Group extensions of Lie groups. I, Ann. of Math. (2) 54 (1951), 96-109.

11. - Group extensions of Lie groups. II, Ann. of Math. (2) 54 (1951), 537-551.

12. G. Hochschild and J.-P. Serre, Cohomology of group extensions, Trans. Amer. Math. Soc. 74 (1953), 110-134.

13. K. Iwasawa, Some types of topological groups, Ann. of Math. (2) 50 (1949), 507-558.

14. J. L. Kelley, General topology, Van Nostrand, New York, 1955.

15. C. Kuratowski, Topologie. I, 3rd ed., Polskie Towarzystwo Matematyczne, Warsaw, 1952.

16. G. W. Mackey; Induced representations of locally compact groups. I, Ann. of Math. (2) 55 (1952), 101-139.

17. - Les ensembles Borélien et les extensions des groupes, J. Math. Pures Appl. 36 (1957), 171-178.

18. — Borel structures in groups and their duals, Trans. Amer. Math. Soc. 85(1957), 134-165.

19. _ Unitary representations of group extensions. I, Acta. Math. 99 (1958), 265-311. 
20. D. Montgomery and L. Zippen, Topological transformation groups, Interscience, New York, 1955.

21. P. S. Mostert, Local cross sections in locally compact groups, Proc. Amer. Math. Soc. 4 (1953), 546-649.

22. H. Nagao, The extension of topological groups, Osaka Math. J. 1 (1949), 36-42.

23. L. Pontrjagin, Topological groups, Princeton, N. J., 1940.

24. A. Shapiro, Group extensions of compact Lie groups, Ann. of Math. (2) 50 (1949), 581-658.

25. H. Weyl, The theory of groups and quantum mechanics, New York, 1931.

26. E. Wigner, On unitary representations of the inhomogeneous Lorentz group, Ann. of Math. (2) 40 (1939), 149-204.

27. G. D. Mostow, Cohomology of topological groups and solvmanifolds, Ann. of Math. (2) 73 (1961), 20-49.

HARVARD UNIVERSiTY,

Cambridge, MassachusetTs

THE UNIVERSITY OF Chicago,

Chicago, Illinois 